\title{
Examining the Effects of Oxygen Plasma on Physical and Dyeing Properties of Some Cellulose Fibers
}

\section{Dilara KOCAK*}

\author{
Marmara University, Technology Faculty, Department of Textile Engineering Goztepe/Istanbul-Turkey \\ cross $^{\text {ref }}$ http://dx.doi.org/10.5755/j01.ms.22.3.9368
}

Received 14 January 2015; accepted 19 June 2015

\begin{abstract}
Cotton, Agava Americana and artichoke fibers were treated with plasma with oxygen gas in Diener Vacuum Plasma for 1,3 and 5 minutes, with $40 \mathrm{kHz}$ low frequency and at 0.3 mbar pressure. After the plasma treatment, fibers' weight loss $\%$, tensile strength, elongation, fiber diameter, surface topography (SEM), colour changes, and light and washing fastness properties were investigated. A positive increase was observed for mechanical and fastness properties after 5 min plasma treatment. The effects of plasma treatments on dyeing properties of fibers were studied. Dyeing properties of plasma treated fibers were improved after 3 min. treatment. SEM results were also proved the improved physical properties and colour changes due to the rough surface structure.

Keywords: cotton, Agava Americana, artichoke fibers, oxygen plasma treatment, dye exhaustion \%, mechanical properties.
\end{abstract}

\section{INTRODUCTION}

Water consumption, waste and environmental pollution are high in traditional textile treatments. Plasma treatments are eco-friendly systems which consume less energy, water and chemicals. Also, they don't change volumetric properties of textile materials [1]. There have also been studies on natural fibers, in which the plasma technology was used in order to give materials water and oil repellent properties. For this purpose, plasma treatment was applied on cotton, wool, and silk using siloxane, perfluorocarbon, and sulphur hexa fluoride and acrylate gases. For cotton and wool, oxygen plasma treatment increased specific surface area as well [2]. While cotton's pilling, thermal resistance and water vapour permeability properties improved with atmospheric pressure plasma, thermal conductivity and air permeability properties decreased [3]. Plasma systems increase the processing efficiency in terms of dyeability and application of printing technologies in textile. The reasons of the increase in efficiency include improvement in capillary system, enlargement of surface area, decrease in excess crystals and formation of reactive groups on fibers [4].

Since 1998, Agava Americana fibers were used as a new textile fiber. These plants are grown in equatorial zone especially in Mediterranean coasts. Agava fibers are extracted from the leaves of certain agava plants. These fibers occur, after their extraction, in bundles constituted of several ultimate fibers held together by waxy and sticky substances. Ultimate fibers have an average equivalent diameter of $24 \mu \mathrm{m}$ and a length of 1 to $7.5 \mathrm{~mm}$, these fibers consist of cellulose 68.42 wt. $\%$, lignin 4.85 wt. $\%$, hemicelluloses 15.67 wt. $\%$, wax content 0.26 wt. $\%$, and moisture content 7.69 wt.\% [5].

Cynara scolymus $L$. is a vegetable, which is a member of Asteraceae family. After the harvest, artichoke's plant body is left as agricultural waste. Artichoke fiber is obtained using the machine designed to obtain fiber from leftover plant body. By the help of the machine, which has cylinder and knife groups, fibers are extracted from the stem of the artichoke and agava plant. Artichoke fiber is a suitable fiber for composite structures. The study shows that artichoke plant's chemical structure consists of $75.3 \%$ cellulose, $4.3 \%$ lignin, and $2.2 \%$ ash. Its tensile strength is $100 \mathrm{MPa}$, elongation is about $2.75 \%$ [6]. The structure of artichoke fiber was studied by many researchers in terms of antioxidative, antimicrobial, and antifungal properties [7].

In this study, plasma treatment was applied on cotton, Agava Americana and artichoke fibers at $40 \mathrm{kHz}$ low frequency and at 0.3 mbar pressure for different durations (1, 3 and 5 minutes). Tensile strength and elongation, colour changes, light and washing fastnesses and morphological properties of fibers, before and after the plasma treatment were examined.

\section{EXPERIMENTAL DETAILS}

\subsection{Materials}

Fibers: cotton, Agava Americana and artichoke fibers were used. The physical properties of the untreated fibers were given in Table 1.

Table 1. Physical properties of untreated fibers

\begin{tabular}{|l|c|c|c|}
\hline \multicolumn{1}{|c|}{ Properties } & Cotton & Agava Americana & Artichoke \\
\hline Diameter, $\mu \mathrm{m}$ & 14.5 & 198.7 & 202.1 \\
\hline Tensile strength, MPa & 267.2 & 478.4 & 321.7 \\
\hline Elongation, \% & 3.71 & 7.98 & 1.84 \\
\hline
\end{tabular}

Dye and Auxiliaries: for the experiment Denkom SYT (Denge Chemistry) which was a commercial product with an anionic-nonionic structure was used. Homobifunctional reactive dye Cibacron Scarlet LS-2G (C.I Reactive Red 268) commercial product was used. This dye was obtained from Ciba and used without being purified. For washing after

\footnotetext{
${ }^{*}$ Corresponding author. Tel.: +902163365770.

E-mail address: dkocak@marmara.edu.tr (D. Kocak)
} 
dyeing, Denwash P5 Denge Chemistry) commercial product, which has an anionic structure, was used.

\subsection{Methods}

\subsubsection{Plasma treatment}

The plasma treatments of fibers were carried out with oxygen gas in Diener Vacuum Plasma. Vacuum plasma is a discontinuous system. Vacuum chamber affects the size of the sample. Researchers have reported that oxygen gas in Diener Vacuum Plasma method was effective on modifying textile materials. The plasma treatment was performed with low frequency, at $40 \mathrm{kHz}$, and the duration of treatment lasted 1, 3 and 5 minutes [8].

\subsection{2. $K / S$ values}

The dyed samples' colors using the colour strength $(K / S)$, calculated using Kubelka-Munk equation were evaluated as:

$K / S=(1-R)^{2} / 2 R$,

Where $R$ is the fibers' reflectance value at maximum absorption wavelength; $K$ is the absorption coefficient; and $S$ is the scattering coefficient [9].

\subsubsection{Dyeing process}

These experiments were performed using a 1:15 (liquor ratio) Ahiba Texomat laboratory dyeing machine. Dye baths to be $1 \%$ (owf) were set starting. $20 \mathrm{gL}^{-1}$ of sodium sulphate and $20 \mathrm{gL}^{-1}$ of sodium carbonate to the bath were added. Bath temperature to $80^{\circ} \mathrm{C}$ in 20 minutes was raised and the temperature for another 40 minutes was kept (repeated 3 times). After the bath was cooled down to $40^{\circ} \mathrm{C}$, the bath was emptied. The samples were neutralized and soaped using $1 \mathrm{gL}^{-1}$ of soap (Denwash P5).

\subsubsection{Determining the dye concentration in the dye bath}

In order to determine the dye concentration in the dye bath, bath's absorbance in every 10 minutes using Colorgen spectrophotometer was measured. 6 similar dyeings were performed for each experiment. One of the 6 dyeings was terminated at $10^{\text {th }}, 20^{\text {th }}, 30^{\text {th }}, 40^{\text {th }}, 50^{\text {th }}$ and $60^{\text {th }}$ minutes. Spectrophotometric measurements were performed at $540 \mathrm{~nm}$ for C.I Reactive Red 268. The dye concentration in the dye-bath and on the fibers was calculated according to the Lambert-Beer law Eq. 2 [9].

$A=k \cdot c \cdot l$,

Where $A$ is the measured absorbance; $k$ is the absorption coefficient of the dye in $\mathrm{lg}^{-1} \mathrm{~cm}^{-1} ; c$ is the concentration of the dye in $\mathrm{gl}^{-1}$ and 1 is the length of the optical path in $\mathrm{cm}$. The absorbance values were used in the calibration equation to find dye concentrations in the float at the minutes mentioned above, and dye uptake $\%$ values were calculated with the help of these data Eq. 3.

Dye uptake $\%=\left[\left(c_{1}-c_{2}\right) / c_{1}\right] \times 100$,

where $c_{1}$ is the initial concentration $\mathrm{gL}^{-1} ; c_{2}$ is the concentration at $\mathrm{x}^{\text {th }}$ minute of the process, $\mathrm{gL}^{-1}$.

Color measurements of dyed samples were performed using Datacolor Spectra Flash 600 plus reflector spectrometer and Datamaster software according to CIEL* $a * b$ system. CIEL*a*b values of the fibers were calculated using $10^{\circ}$ standard observer value and D65 illuminant. For measurements, the samples dyed without using plasma treatment were taken as standard and dyeings were evaluated in terms of lightness (L*). CIEL*a*b. 1976 colour differences formula was used to express the colour differences $\left(\Delta \mathrm{L}^{*}\right)$. Calculating colour of dyed samples how darker $\left(-\mathrm{L}^{*}\right)$ or brighter $\left(+\mathrm{L}^{*}\right)$ were compared than the standard.

\subsubsection{Fastness tests}

The light fastness tests were carried out in accordance with the method described in ISO 105-B02. The washing fastness tests were performed in accordance with the method described in ISO 105-C06. The rubbing fastness tests were examined in accordance with ISO 105 X12.

\subsubsection{Characterization}

Before performing the characterization tests, fiber examples at $20^{\circ} \mathrm{C}$ and $65 \%$ relative humidity for at least 24 hours were conditioned. A sample of 100 fibers was randomly taken from fibers, and the diameters were measured by using the microscobic method. In these fibers the diameter varies throughout the length of the fiber (three different points as top, mid-span, and tail), and fiber diameter $(\mu \mathrm{m})$ properties were measured with a Projectina CH-9495 model microscope. Fifty measurements were performed, 10 fibers were calculated from 5 different locations along the fiber. Tensile strength and elongation properties of the fibers were tested according to ASTM D 3822 by using Instron 4411 Model.

\subsubsection{Weight loss}

The weight loss was determined on atmospherically conditioned fibers after different treatment processes. The weight loss percentage $\left(W_{j}\right)$ was calculated from the differences in weight using the following equation:

$W j=\frac{\text { Wpre }- \text { Wafter }}{\text { Wpre }} \times 100(\%)$,

where $W_{\text {pre }}$ is the weight of the conditioned fibers prior to pre-treatment and $W_{\text {after }}$ is the weight after performed pretreatment.

\subsubsection{Morphological properties}

The morphological (SEM) properties of samples were analysed with JEOL JSM-T330 instrument, which was operated at $5 \mathrm{kV}$ and $10 \mathrm{kV}$, according to TS EN ISO 9220 standard. The samples were coated with gold in order to have an increase in the conductivity.

\section{RESULTS}

\subsection{Fiber diameter}

Table 2 presents the average values, standard deviation (SD) and coefficient of variation (CV), of untreated and plasma treated cotton, Agava Americana and artichoke fibers. These fibers characteristically have higher SD and $\mathrm{CV}$ values. 
Table 2. Comparison of the fiber diameter of untreated and plasma treated fibers

\begin{tabular}{|c|c|c|c|c|c|c|c|c|c|}
\hline Samples & \multicolumn{3}{|c|}{ Cotton fiber } & \multicolumn{3}{|c|}{ Agava Americana fiber } & \multicolumn{3}{|c|}{ Artichoke fiber } \\
\hline Diameter, $\mu \mathrm{m}$ & Mean & SD & $\mathrm{CV}$ & Mean & SD & $\mathrm{CV}$ & Mean & SD & $\mathrm{CV}$ \\
\hline Untreated & 14.5 & 32.4 & 15.7 & 188.7 & 32.7 & 19 & 202.1 & 47 & 13.2 \\
\hline Plasma Treatment $1 \mathrm{~min}$ & 14.3 & 15.3 & 15.1 & 180.4 & 20.4 & 21 & 187.3 & 26 & 11.2 \\
\hline Plasma Treatment $3 \mathrm{~min}$ & 13.7 & 27.3 & 38.2 & 163.5 & 18.4 & 18 & 161.3 & 12 & 15.6 \\
\hline Plasma Treatment 5min & 12.9 & 29.4 & 40.1 & 151.7 & 13.7 & 16 & 131.8 & 15 & 10.6 \\
\hline
\end{tabular}

Fiber diameter changes on treated fibers decreased significantly when compared to untreated fibers. Cotton fibers' diameter decreased from $14.5 \mu \mathrm{m}$ to $12.9 \mu \mathrm{m}$. The decrease percentage was $11 \%$. For Agava Americana fibers it was $32.4 \%$, and for artichoke fibers it was $34.8 \%$. A thinning has occurred in fiber diameters. Thinning amount was greater in Agava Americana and artichoke fibers when compared to cotton, since they had more lignin. It's thought that plasma treatment was effective on lignin layer, which has a non-cellulosic structure. Penetration of plasma's ionized gases into fibers was effective on diameter thinning [10].

\subsection{Weight loss \%}

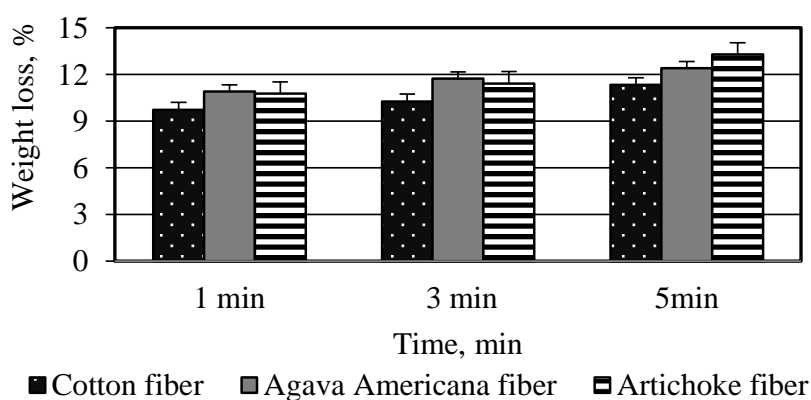

Fig. 1. Change in weight loss behaviour as a result of plasma treatment

Fig. 1., shows the change in weight loss $\%$ values of cotton, Agava Americana and artichoke fibers after the plasma treatment with different durations. Weight loss values increased depending on durations for cotton fiber $16.3 \% \quad(9.7 \% / 1 \mathrm{~min}-11.3 \% / 5 \mathrm{~min}), \quad$ for Agava Americana fibers $23.9 \%(10.92 \% 1 \mathrm{~min}-12.7 \% 5 \mathrm{~min})$, for artichoke $20.4 \%(10.78 \% / 1 \mathrm{~min}-12.9 \% / 5 \mathrm{~min})$. It has been reported that weight loss of the plasma treated fiber was essentially due to the cleaning of the surface contaminants and etching of the fiber. Plasma treatment reacted the fibers' hydroxyl groups. The reason for such observation was the removal of residual size materials and impurities from the fibers during plasma treatment. Also, lignocellulosic fibers (Agava Americana and artichoke fibers) had higher content of impurities than cotton fiber due to their lignin [11].

\subsection{Mechanical properties}

Results of tensile strength of each different sample were shown in Fig. 2. There was an increase in tensile strength about $6.5 \% / 1 \mathrm{~min}, 9.4 \% / 3 \mathrm{~min}, 14 \% / 5 \mathrm{~min}$ in cotton fibers; $1.5 \% / 1 \mathrm{~min}, 3.2 \% / 3 \mathrm{~min}, 4.9 \% / 5 \mathrm{~min}$ in Agava Americana fibers and $2.2 \% / 1 \mathrm{~min}, 2.6 \% / 3 \mathrm{~min}$, $3.5 \% / 5 \mathrm{~min}$ in artichoke fibers. Depending on the duration, the tensile strength of all fibers increased. The oxygen plasma was effective on fibers' properties at different physical and chemical conditions. The oxygen plasma changed the physical properties of fibers and more in particular their increase in tensile strength positively [12].

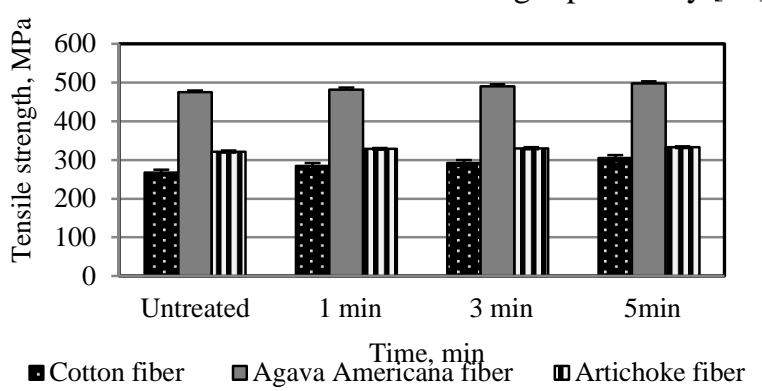

Fig. 2. Change in tensile strength behaviour as a result of plasma treatment

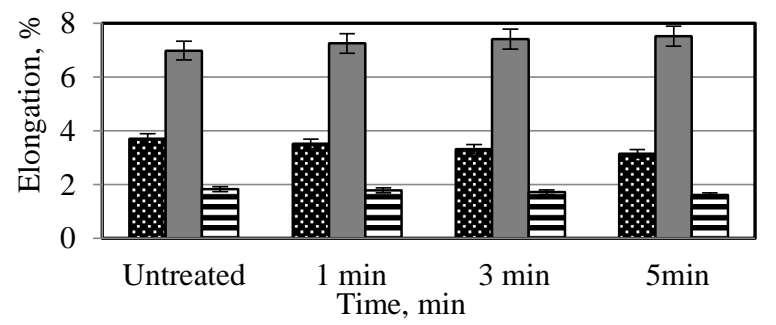

Cotton fiber $\square$ Agava Americana fiber $\square$ Artichoke fiber

Fig. 3. Change in elongation behaviour as a result of plasma treatment

As a result of the plasma treatment, there was decrease in elongation $\%$ values of fibers related to increase in duration as shown in Fig. 3. There were changes in elongation about $5 \% / 1 \mathrm{~min}, 11 \% / 3 \mathrm{~min}, 15 \% / 5 \mathrm{~min}$ in cotton fibers; $8 \% / 1 \mathrm{~min}, 11 \% / 3 \mathrm{~min}, 12 \% / 5 \mathrm{~min}$ in Agava Americana fibers; and $3 \% / 1 \mathrm{~min}, 7 \% / 3 \mathrm{~min}, 12 \% / 5 \mathrm{~min}$ in artichoke fibers. This can be explained by the increased interaction between fibers after plasma treatment, which caused reduction in effective gap between fibers at their crossover points and lateral compressional abilities of the microfibrils in fibers, leading to reduction of extensibility [13].

\subsection{Dyeing properties of fibers}

Plasma treatments were applied on cotton, Agava Americana and artichoke fibers at different durations (1, 3 and $5 \mathrm{~min}$ ), and then they have been dyed with reactive dye. Dye uptake curves and relative final absorption values of cotton fibers, which was plasma treated at different durations, are shown in Fig. 4.

Fig. 4a., shows that dye uptake values of untreated cotton fibers were less than plasma treated samples $(76.3 \%)$. The highest dye uptake value was obtained in 3 minute (87\%) plasma treated dyed cotton fibers. Relative final exhaustion values of untreated cotton (100), 1 min plasma treated cotton (112.6), 3 min plasma treated cotton 
(113.9) and 5 min plasma treated cotton (112.7) are given in Fig. 4b. For the untreated and plasma treated dyed cotton fibers $K / S$ values are $1.61,1.87,2.17,1.71$ respectively as shown in Table 3 . The highest $K / S$ value was obtained in 3 minute (2.17).
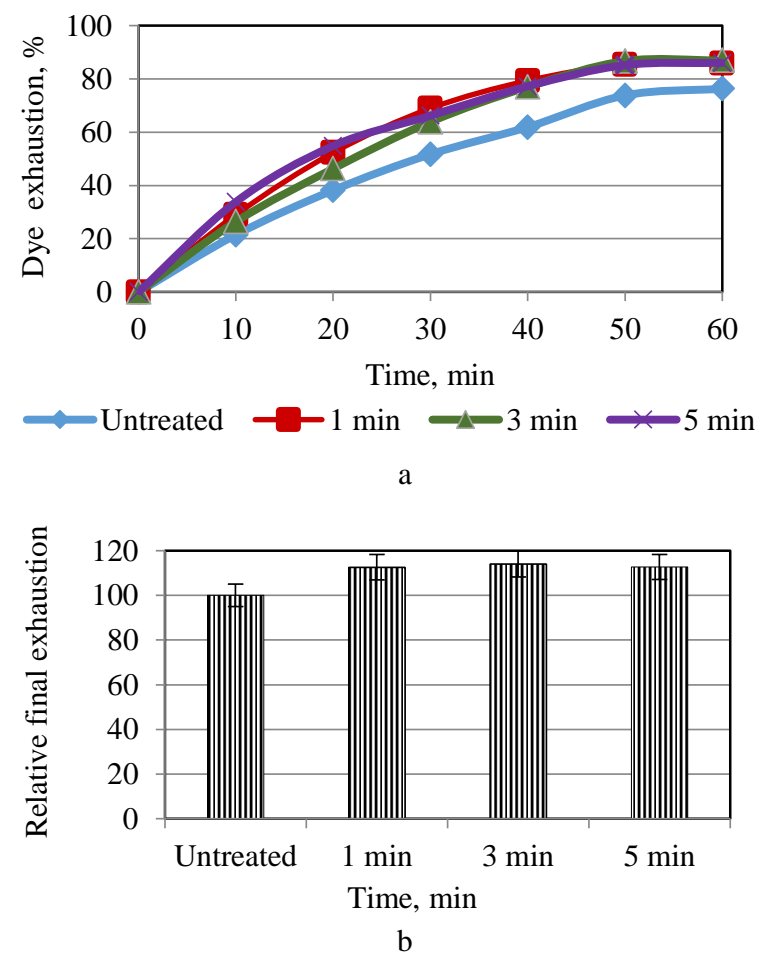

Fig. 4. Dye uptake $\%$ values of cotton fibers: a - comparison of dye uptake values of cotton fibers; $b$-comparison of relative final exhaustion values of cotton fibers

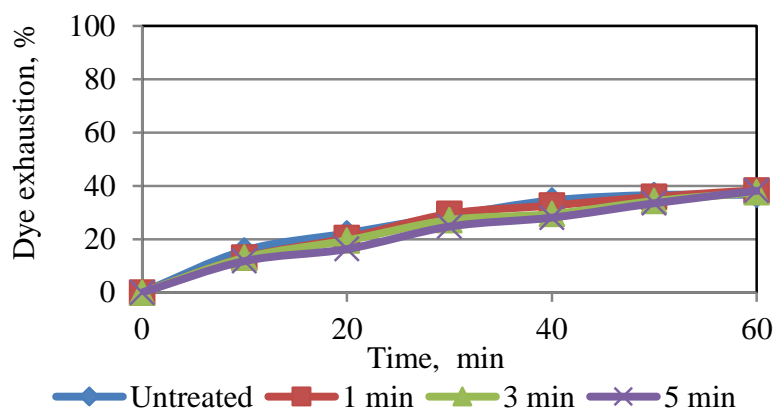

a

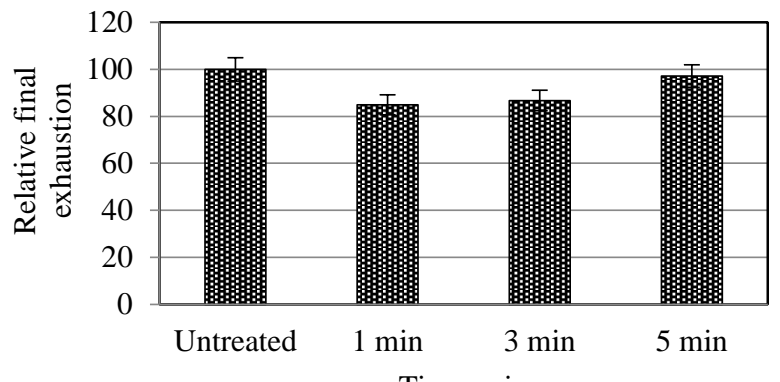

Time, $\min$

b

Fig. 5. Dye uptake \% values of Agava Americana fibers: a-comparison of dye uptake values of Agava Americana fibers; $\mathrm{b}$ - comparison of relative final exhaustion values of Agava Americana fibers
Dye uptake curves and relative final absorption values of Agava Americana fibers, which is plasma treated at different durations, were shown in Fig. 5. Fig. 5a shows that dye uptake values of untreated Agava Americana fibers were less than 5 min plasma treated samples $(51.7 \%)$. Dye uptake values of the Agava Americana fibers increased by the increasing of durations, the highest dye uptake value was obtained in 5 minute $(50.2 \%)$ for plasma treated dyed Agava Americana fibers. Relative final exhaustion values of untreated Agava Americana (100), 1 min plasma treated Agava Americana (84.8), 3 min plasma treated Agava Americana (86.7) and $5 \mathrm{~min}$ plasma treated Agava Americana fibers (97.1) are given in Fig. 5b. For the untreated and plasma treated dyed Agava Americana fibers, $K / S$ values are $2.8,2.2,2.5,3.5$ respectively as shown in Table 3. The highest $K / S$ value was obtained in 5 minute (3.5).
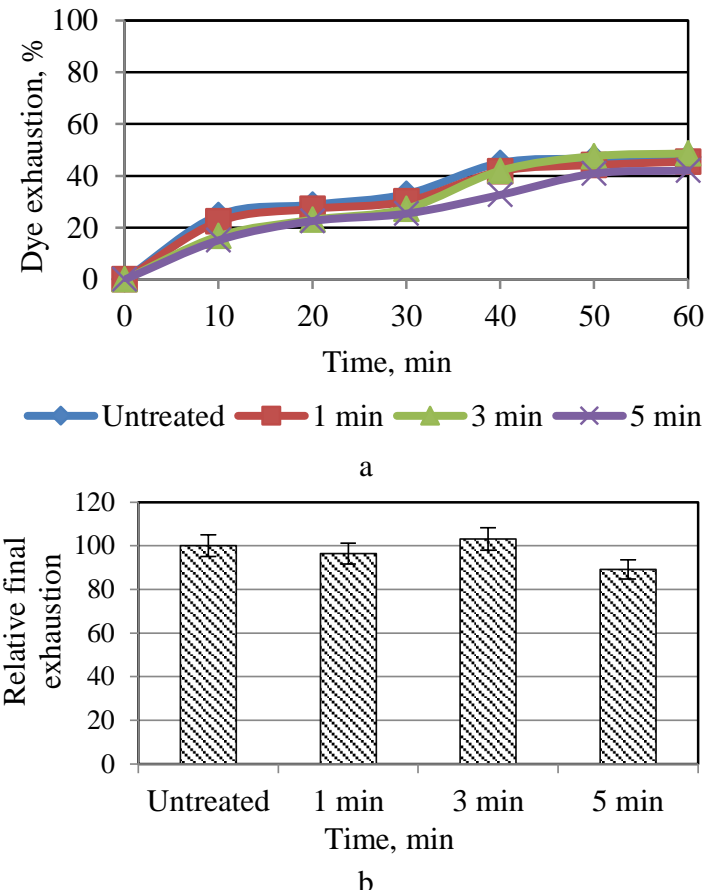

Fig. 6. Dye uptake $\%$ of artichoke fibers: a-comparison of dye uptake values of artichoke fibers; $b$-comparison of relative final exhaustion values of artichoke fibers

Dye uptake curves and relative final absorption values of artichoke fibers, which was plasma treated at different durations, are shown in Fig. 6. Fig. 6 a shows that dye uptake values of untreated artichoke fibers were less than plasma treated samples $(47.1 \%)$. The highest dye uptake value was obtained in 3 minute $(48.6 \%)$ for plasma treated dyed artichoke fibers. Relative final exhaustion values of untreated artichoke (100), 1 min plasma treated artichoke (96.4), 3 min plasma treated artichoke (103.1) and 5 min plasma treated artichoke fibers (89.2) are given in Fig. 6 b. For the untreated and plasma treated dyed artichoke fibers, $\mathrm{K} / \mathrm{S}$ values are $1.29,1.21,1.31,1.10$ respectively as shown in Table 3. There was no important change in K/S values of untreated, plasma treated and dyed artichoke fibers. The reason of the better dyeing properties of the treated and dyed samples was the hydrophobic outer layers of plasma treated fibers, which were removed to allow the dye penetrating more easily. 
Table 3. CIELab colour differences and values of undyed and plasma treated dyed samples

\begin{tabular}{|c|c|c|c|c|c|c|c|c|c|c|c|c|}
\hline Material & $\mathrm{L}^{*}$ & $a^{*}$ & $b^{*}$ & $\mathrm{C}^{*}$ & $h^{\circ}$ & $\mathrm{K} / \mathrm{S}(550 \mathrm{~nm})$ & $\Delta \mathrm{L}^{*}$ & $\Delta \mathrm{a}^{*}$ & $\Delta \mathrm{b}^{*}$ & $\Delta \mathrm{C}^{*}$ & $\Delta \mathrm{E}^{*}$ & $\Delta \mathrm{H}^{*}$ \\
\hline $\bar{A}$ & 63.4 & 37.9 & -9.6 & 39.1 & 345.8 & 1.61 & - & - & - & - & - & - \\
\hline $\mathrm{A} 1$ & 62.7 & 41.8 & -9.7 & 42.9 & 347.0 & 1.87 & -0.735 & 3.873 & -0.24 & 3.770 & 3.942 & 0.888 \\
\hline $\mathrm{A} 2$ & 60.9 & 43.0 & -9.6 & 44.1 & 347.4 & 2.17 & -2.540 & 5.145 & 0.014 & 5.001 & 5.738 & 1.205 \\
\hline A3 & 63.9 & 41.4 & -9.3 & 42.4 & 347.3 & 1.71 & 0.461 & 3.512 & 0.307 & 3.344 & 3.556 & 1.116 \\
\hline $\mathrm{B}$ & 55.8 & 38.0 & -4.2 & 38.3 & 353.8 & 2.76 & - & - & - & - & - & - \\
\hline $\mathrm{B} 1$ & 58.9 & 37.7 & -4.5 & 37.9 & 353.2 & 2.20 & 3.123 & -0.358 & -0.335 & -0.318 & 3.161 & 0.373 \\
\hline $\mathrm{B} 2$ & 57.0 & 38.4 & -4.4 & 38.6 & 353.5 & 2.55 & 1.162 & 0.330 & -0.228 & 0.353 & 1.230 & 0.190 \\
\hline B3 & 53.1 & 40.5 & -5.6 & 40.9 & 352.1 & 3.46 & -2.670 & 2.480 & -0.480 & 2.644 & 3.933 & 1.162 \\
\hline $\mathrm{C}$ & 62.7 & 24.6 & 0.5 & 24.6 & 1.1 & 1.29 & - & - & - & - & - & - \\
\hline $\mathrm{C} 1$ & 64.3 & 26.9 & -1.3 & 26.9 & 357,3 & 1.21 & 1.565 & 2.252 & -0.757 & 2.278 & 3.257 & 1.722 \\
\hline $\mathrm{C} 2$ & 63.0 & 25.6 & 1.40 & 25.7 & 3.1 & 1.29 & 0.307 & 1.000 & 0.931 & 1.034 & 1.401 & 0.894 \\
\hline $\mathrm{C} 3$ & 65.1 & 25.2 & -0.1 & 25.2 & 359.9 & 1.10 & 2.406 & 0.586 & -0.519 & 0.581 & 2.530 & 0.524 \\
\hline \multicolumn{13}{|c|}{$\begin{array}{l}\text { A: Untreated cotton fiber, A1:1 min plasma treated cotton fibers, A2:3 min plasma treated cotton fibers A3: } 5 \text { min plasma treated cotton fibers, } \\
\text { B: Untreated Agava Americana fiber B1: } 1 \text { min plasma treated Agava Americana fibers, B2: } 3 \text { min plasma treated Agava Americana fibers, B3: } 5 \text { min } \\
\text { plasma treated Agava Americana fibers, } \\
\text { C: Untreated artichoke fiber, C1:1 min plasma treated artichoke fiber, C2: } 3 \text { min plasma treated, artichoke fiber, C3: } 5 \text { min plasma treated artichoke } \\
\text { fiber. } \\
\text { (The huge difference between the batch and the standard is defined as } \Delta H^{*}=\left[\left(\Delta E^{*}\right) 2-\left(\Delta L^{*}\right)^{2}-\left(\Delta C^{*}\right)^{2}\right]^{1 / 2} ; \Delta H^{*} \text { is to be regarded as 'positive' if } \\
\text { indicating an increase in hab and 'negative' if indicating a decrease). }\end{array}$} \\
\hline
\end{tabular}

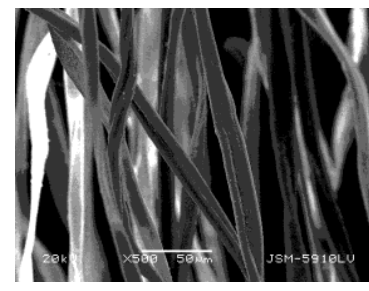

a

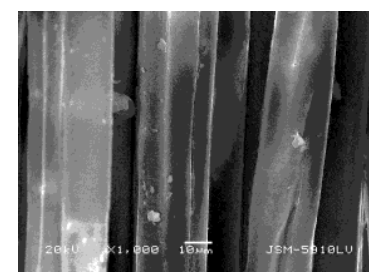

c

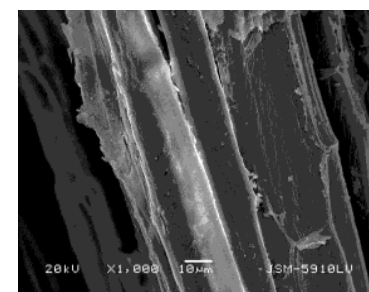

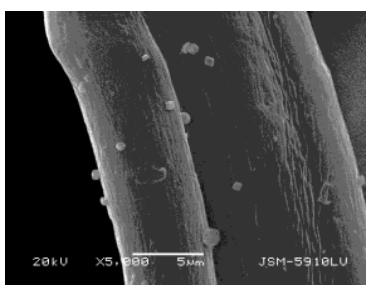

b

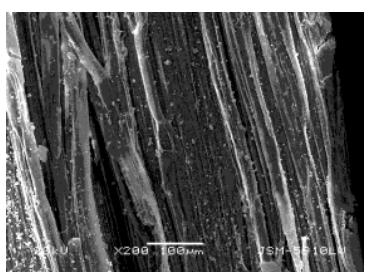

d

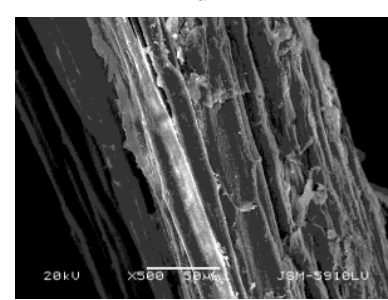

f
Fig. 7. SEM images of plasma treated and untreated cotton fibers: $a-$ untreated cotton fibers; $b$ - plasma treated cotton fibers (5 min); c-untreated Agava Americana fibers; d-plasma treated Agava Americana fibers (5 min); e-untreated artichoke fibers; $\mathrm{f}-$ plasma treated artichoke fibers $(5 \mathrm{~min})$

\subsection{Fastness values}

The light fastnesses of the dyed fiber samples, with the help of plasma treatment, were quite better than all untreated fibers. The colour change of all samples, after the washing fastness tests, was quite good. When staining results were compared, poor staining on polyester (level 4-5) was determined. The other staining results were good. The colour fastness to dry and wet rubbing values of all fiber samples dyed with help of plasma treatment were good (level 4 and $4-5+$ ). The best results of duration dependant fastness value changes are obtained with plasma treatment for 5 minutes. As a result of plasma treatment, the fastness properties of the samples were increased.

\subsection{SEM analysis}

The surface topographies of untreated cotton fibers are shown in Fig. 7 a. The surface topographies of 5 minutes plasma treated cotton fibers are shown in Fig. $7 \mathrm{~b}$. The surface topographies of untreated Agava Americana fibers are shown in Fig. 7 c. The 5 minutes plasma treated Agava Americana fibers are shown in Fig. $7 \mathrm{~d}$. The surface topographies of untreated artichoke fibers are shown in Fig. 7 e. The surface topographies of 5 minutes plasma treated artichoke fibers are shown in Fig. $7 \mathrm{f}$. For all fibers after the 5 minutes plasma treatment, the surface was peeled when compared to untreated fibers. During oxygen plasma bombardment onto the fiber surfaces, the holes have been created allowing the dye to infuse better. Roughness was higher on Agava Americana than on artichoke fiber surfaces. The reason was the oxygen plasma penetrating the fiber surface and removing wastes such as lignin, oil and wax from the fiber surface. For this reason, the surface topography was rough. Oxygen plasma caused a drastic reduction in surface resistivity of Agava Americana and artichoke fibers. For cotton fibers, oxygen bombardment created roughness on the surface and as for other fibers; this allowed dye to diffuse better [14].

\section{CONCLUSIONS}

The advantages of using oxygen plasma treatment can be summarised as follows:

1. 5 minutes plasma treatment was effective on weight loss values.

2. Oxygen used in plasma treatment improved fibers' tensile strength and elongation properties. Oxygen gas induced in the fibers and affected fibers' molecular structure.

3. An increase in plasma treatment duration resulted as a positive effect on whiteness index for all the fibers. 
4. This technique increases the dye uptake of cotton fibers, and dyeing with reactive dyes can help to obtain better colouristic and fastness properties. The high colour yield $(K / S)$ was obtained in 5 minutes plasma treated Agava Americana fibers. The dye uptake results of cotton fibers were higher than artichoke and Agava Americana fibers due to less lignin content. The plasma treatment is effective on improving light, washing and rubbing fastness properties of dyeings.

5. Functional groups formed by oxygen plasma were interacted with fiber structure, and this can increase the fixing rate of dye molecules into the fiber.

6. SEM results proved the improved physical properties and colour changes due to the rough surface structure.

7. As a result, oxygen plasma treatment process is effective on physical and dyeing properties of fibers.

\section{REFERENCES}

1. Sparavigna, A. Plasma Treatment Advantages for Textiles Popular Physics http://arxiv.org/abs/0801.3727:2007.

2. Ferrero, F. Wettability Measurements on Plasma Treated Synthetic Fabrics by Capillary Rise Method Polymer Testing 22 (5) 2007: pp. $571-578$.

3. Höcker, H. Plasma Treatment of Textile Fibers Pure Applied Chemistry 74 (3) 2002: pp. 425-427.

4. Karahan, H.A. at al Effects of Atmospheric Pressure Plasma Treatments on Certain Properties of Cotton Fabrics Fibres \& Textiles in Eastern Europe 17 (2-73) 2009: pp. 19-22.

5. Fiore, V., Valenza, A., Di Bella, G. Artichoke (Cynara Cardunculus L.) Fibres as Potential Reinforcement of Composite Structures Composites Science and Technology 71 2011: pp. $1138-1144$.
6. Fritsche, J. Isolation, Characterization and Determination of Minor Artichoke (Cynara Scolymus L.) Leaf Extract Compounds European Food Research and Technology 215 (2) 2002: pp. $149-157$. http://dx.doi.org/10.1007/s00217-002-0507-0

7. Emanuel, V. at al. Antioxidant and Antimicrobial Activities of Ethanol Extracts of Cynara Scolymus (Cynarae Folium, Asteraceae Family) Tropical Journal of Pharmaceutical Research 10 (6) 2011: pp. 777-783.

8. Sparavigna, A. Plasma Treatment Advantages for Textiles http://arxiv.org/ftp/arxiv/papers/0801/0801.3727. Pdf 2015

9. Baltazar, A., Bistritz, J.M., Schulz, E., Bismarck, A. Atmospheric Air Pressure Plasma Treatment of Lignocellulosic Fibres: Impact on Mechanical Properties and Adhesion to Cellulose Acetate Butyrate Composites Science and Technology 68 (1) 2008: pp. 215-227.

10. Gorjanc, M., Bukošek, V. Gorenšek, M., Vesel, A. The Influence of Water Vapor Plasma Treatment on Specific Properties of Bleached and Mercerized Cotton Fabric Textile Research Journal 82 2004: pp. 62-69.

11. Sghaier, A.Q.B., Chaabouni, Y., Msahli, S., Sakli, F. Morphological and Crystalline Characterization of $\mathrm{NaOH}$ and $\mathrm{NaOCl}$ Treated Agava Americana L. Fiber Industrial Crops and Products 36 (1) 2012: pp. 257-266. http://dx.doi.org/10.1016/j.indcrop.2011.09.012

12. Wong, K.K., Tao, X.M., Yuen, G.W.M., Yeung, K.M. Low Temperature Plasma Treatment of Linen Textile Research Journal 69 (11) 2009: pp. 846-855.

13. Broadbent, A.D. Basic Principles of Textile Coloration Society of Dyers and Colourists 2001: pp. 477-482.

14. Karahan, H.A., Ozdogan, E., Demir, A., Ayhan, H., Seventekin, N. "Effects of Atmospheric Plasma Treatment on The Dyeability of Cotton Fabrics by Acid Dyes Coloration Technology (124) 2008: pp. 106-110. 